\title{
Molekulares Verstehen des Hörens - Was ändert sich für den Patienten?
}

\section{Molecular Understanding of Hearing - How Does This Matter to the Hearing Impaired?}

\section{(ㄷ) (1) (요 $\ominus$}

\author{
Autor
}

Tobias Moser

Institut

Institut für Auditorische Neurowissenschaften, Universitätsmedizin Göttingen

Schlüsselwörter

Cochlea, Schwerhörigkeit, Genetik, Molekularer Mechanismus, Tiermodell, Therapie

Key words

Cochlea, molecular mechanism, animal model, genetics, deafness, therapy

\section{Bibliografie}

DOI https://doi.org/10.1055/s-0043-121595

Laryngo-Rhino-Otol 2018; 97: S214-S222

(c) Georg Thieme Verlag KG Stuttgart · New York

ISSN 1615-0007

Korrespondenzadresse

Prof. Dr. med. Tobias Moser

Institut für Auditorische Neurowissenschaften

Universitätsmedizin Göttingen

Robert-Koch-Str. 40

D-37075 Göttingen

tmoser@gwdg.de

\section{ZUSAMMENFASSUNG}

Das Innenohr und die Hörbahn mit ihren vergleichsweise geringen Zellzahlen haben sich einigen molekularen Ansätzen bislang beharrlich entzogen. Gleichzeitig vollbringt das Hören Spitzenleistungen, die sehr spezialisierte biologische Mechanismen nahelegen. Dies bedeutet einerseits, dass Analogieschlüsse zur molekularen Anatomie und Physiologie der Zellen des Hörsystems auf der Grundlage von Erkenntnissen aus molekular besser zugänglichen Systemen von beschränktem Nutzen sind. Andererseits legt eine solche Spezialisierung Gendefekte nahe, die von der Evolution toleriert wurden, weil sie nicht zur Fehlfunktion von essentiellen Körperprozessen führen. Technologische Fortschritte in der Humangenetik und der molekularen Analyse des Innenohrs im Tier bestätigen beide Annahmen und beleuchten den faszinierenden Mikrokosmos der Cochlea. Auf kleinstem Raum werden hier in konsequenter Arbeitsteilung herausragende Leistungen im lonentransport, der Mechanotransduktion, der aktiven Zellmotilität und der synaptischen Verarbeitung erbracht. Einige der zugrundeliegenden molekularen Maschinen, z. B. das
Motorprotein Prestin und das an synaptischer Fusion beteiligte Otoferlin, sind ausschließlich im Ohr aktiv. Dementsprechend führen ihre Defekte zu spezifischen nicht-syndromalen Schwerhörigkeiten, wie etwa bei der auditorischen Synaptopathie durch autosomal rezessive Mutationen im Otoferlin-Gen. Andere Mutationen, wie die den cochleären Kalium-Zyklus betreffenden, bedingen einen globalen Funktionsverlust der Cochlea. Viele genetische Defekte führen schließlich zur Degeneration des Innenohrs. Letztlich führt die molekulare Analyse sowohl beim Menschen, als auch im Tier-Innenohr aber auch zu neuen Erkenntnissen für häufige Formen der Schwerhörigkeit. So wurde der immunhistochemische Nachweis des Verlusts von Bandsynapsen der inneren Haarzellen zum Biomarker für „hidden hearing loss“ im Tiermodell. Die moderne HochdurchsatzSequenzierung (sog. Next Generation Sequencing - NGS) bietet Zugang zu bislang nicht bekannten Taubheitsgenen, Mutationsspektren von bekannten Taubheitsgenen und zu einem genetischen Profil der individuellen Schwerhörigkeit, ihre Interpretation erfordert jedoch große humangenetische Expertise und umfangreiche tierexperimentelle Einsichten. Eine kausale Therapie etwa durch viralen Genersatz, der im Tier-Innenohr und bei einzelnen Formen der humanen Blindheit bereits erfolgreich ist, steht für die Schwerhörigkeit in der Klinik noch nicht zur Verfügung. Bereits jetzt ermöglichen molekulare Ansätze aber schon eine verbesserte Beratung von schwerhörigen Patienten.

\section{ABSTRACT}

This review addresses the advances of our molecular understanding of hearing and how this benefits the hearing impaired. Classical biochemical methods usually fall short in contributing to the analysis of the molecular mechanisms of hearing e. $g$. in the cochlea, the auditory part of the inner ear, due to the scarcity of the cells of interest. Genetics, molecular cell biology, and physiology, on the other hand, have elucidated the intricate molecular and cellular mechanisms that bring about the outstanding performance of the auditory system. Many of those mechanisms are quite unique and specialized to serve the specific needs of hearing. Hence, their defects often spare other organs and lead to specific non-syndromic deafness. High throughput sequencing can reveal causes of sporadic deafness when combined with careful bioinformatics. Molecular approaches are also helpful for understanding more common forms of hearing impairment such as noise-induced hearing impairment. While molecular therapies are not yet clinically available, careful molecular genetic analysis helps to counsel the hearing impaired subjects. 


\section{Inhaltsverzeichnis}

Zusammenfassung

Abstract

1. Von der Humangenetik zum molekularen Mechanismus und zurück zum Patienten

2. Der Kaliumkreislauf, die Transduktion und die Arbeitsteilung in der Cochlea

3. Die cochleäre Verstärkung und die synaptische Schallkodierung

4. Zusammenfassung

5. Danksagung

Literatur

\section{Von der Humangenetik zum}

\section{molekularen Mechanismus und zurück}

\section{zum Patienten}

In den vergangenen Jahren sind zahlreiche Wege zur Aufdeckung von Genen mit wichtiger Funktion im Innenohr beschritten worden. Von der Klonierung aus cDNA-Bibliotheken, die aus tierischem Gewebe zu verschiedenen Zeitpunkten der Innenohr-Entwicklung erzeugt wurden, zur Analyse des Transkriptoms einzelner Zellen, von Proteomics von massiven Kollektionen tierischer Innenohren zu sensitivster Massenspektrometrie immunaufgereinigter subzellulärer Kompartimente, von Kopplungsanalysen in großen oft konsanguinen Familien mit genetischer Schwerhörigkeit bis zur Identifizierung pathogener Mutationen bei sporadischer Schwerhörigkeit mittels neuer Hochdurchsatz-Sequenziertechnologien. Die humangeneti-

schen Ansätze hatten und haben dabei einen besonderen Stellenwert. Zahlreiche Gene mit Schlüsselfunktionen in der Cochlea wurden durch humangenetische Analysen als sog. „Taubheitsgene“ identifiziert (http://hereditaryhearingloss.org/), wobei initial v.a. Kopplungsanalysen mit nachfolgender Sanger-Sequenzierung von Kandidatengenen in der gekoppelten Region [1,2] und nun zunehmend Hochdurchsatz-Sequenzierungsverfahren [3,4] (Next Generation Sequencing [NGS]) verwendet werden ( $\triangleright$ Abb. 1). Anders als bei der früher üblichen auf Mutationen weniger Gene beschränkten Sanger-Sequenzierung (wie z. B. für das GJB2 Gen, das für Connexin 26 kodiert) können hier für die Identifizierung von ursächlichen Mutationen entweder NGS-basierte Panels mit zahlreichen Taubheitsgenen getestet oder gar eine Sequenzierung des gesamten Exoms (Whole Exom Sequencing [WES], alle zur Transkription genutzten Genabschnitte [Exone]) des Schwerhörigen bzw. ein Trio-WES (Patient und Eltern) durchgeführt werden. Die Entscheidung, welche Art der molekularen Diagnostik durchgeführt werden kann und wie viele Taubheitsgene sequenziert werden, wird durch den Kontext der Untersuchung (im Rahmen wissenschaftlicher Untersuchungen oder in der Routinediagnostik) maßgeblich bestimmt.

Zusammen mit dem audiometrischen Phänotyp identifiziert die NGS-basierte Sequenzierung dann auch den Erbgang: z. B. autosomal rezessive (Gen/Erkrankung dann mit DFNB gekennzeichnet, beide Eltern sind Merkmalsträger aber gesund), autosomal dominant (DFNA, je nach Penetranz ist auch eines der Eltern schwerhörig), und X-chromosomal (DFNX, Mütter sind Merkmalsträger, aber meistens gesund, männliche Nachkommen sind betroffen). Die durch NGS-basierte Methoden generierten Sequenzen (sog. „Sequenzierschnipsel“ oder „Reads“) benötigen eine sorgfältige bioinformatische Analyse sowie eine fachgerechte Bewertung gefundener Varianten durch den Humangenetiker, um die potentiell pathogenen Mutationen zu identifizieren. Auch wenn die Sequenzierung

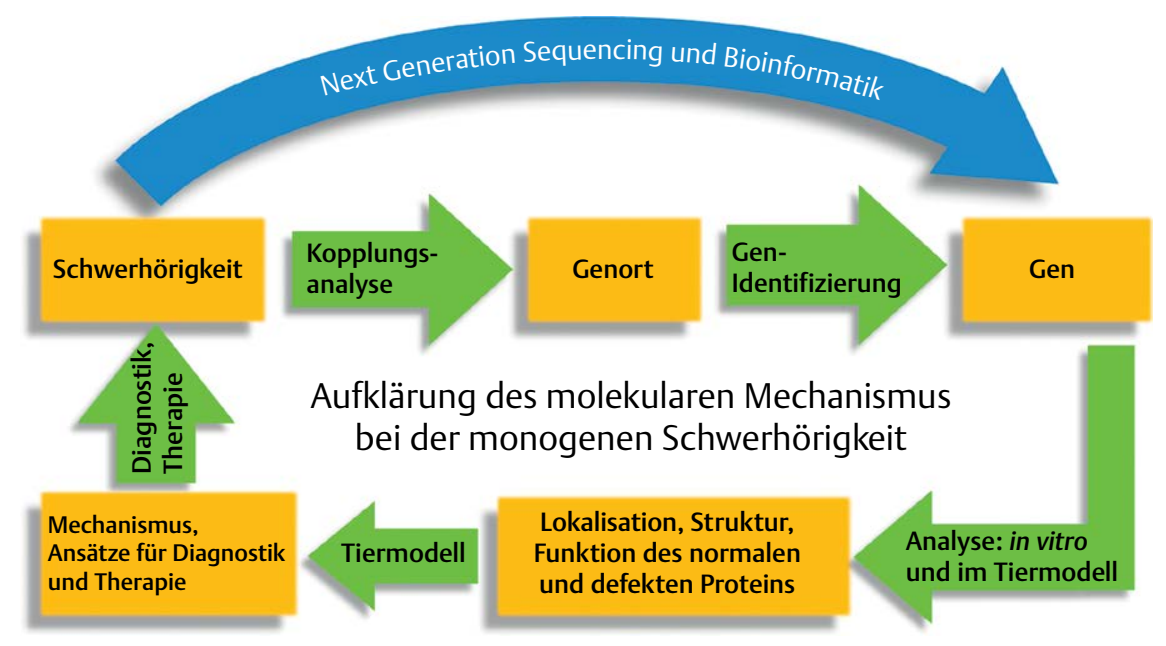

- Abb. 1 Aufklärung des molekularen Mechanismus bei der monogenen Schwerhörigkeit. Bei Anhalt für eine erblich bedingte Schwerhörigkeit kann durch Kopplungsanalyse der Genort des betroffenen Genes, durch Genidentifizierung das betroffene Gen selbst aufgedeckt werden. Dieser Vorgang kann durch Next Generation Sequencing und bioinformatische Methoden wesentlich verkürzt werden. Durch in vitro Analysen und Generierung von Tiermodellen für die entdeckte Mutation können dann Lokalisation, Struktur und Funktion nicht nur des normalen sondern auch des defekten Proteins aufgeklärt werden. Dies erlaubt eine weitere Untersuchung des Krankheitsmechanismus und die Entwicklung von Ansätzen für Diagnostik und Therapie, welche dann zu einer Behandlung der Schwerhörigkeit im Patienten genutzt werden können. 
nun dem informierten HNO-Arzt zugänglich ist, erfordert die Bewertung von Varianten aus NGS-Daten viel Erfahrung und wird daher z. B. im Institut für Humangenetik der UMG in Göttingen durch ein ganzes Expertenteam durchgeführt. Seit Juli 2016 gibt es nun erstmalig EBM-Ziffern, die eine Anwendung NGS-basierter Routinediagnostik erlauben, allerdings gibt es Einschränkungen bei der Anzahl zu analysierenden Gene, sodass bei großen Panels mit vielen Genen oder gar Exomsequenzierungen vorab ein Antrag auf Kostenübernahme bei der entsprechenden Krankenkasse gestellt werden muss. Trotz dieser Einschränkungen stellt die Abrechenbarkeit NGS-basierter Leistungen einen großen Fortschritt dar und wird die Identifizierung pathogener Mutationen bei sporadischer sensorineuraler Schwerhörigkeit erleichtern. Bereits jetzt wird NGS-basierte Analyse umfangreich in der hochschulmedizinischen aber auch in der niedergelassenen Humangenetik genutzt und so empfiehlt es sich hier eine entsprechende Zusammenarbeit aufzubauen.

An die Gen-Identifizierung schließen sich dann die Generierung von molekularen Sonden wie Antikörpern, in vitro struktur- und zellbiologische Analysen des rekombinanten Proteins und die Erstellung von genetisch veränderten Organismen in Fruchtfliege, Zebrafisch und/oder Maus für die weitere funktionelle Charakterisierung des Gens an [5]. Dabei werden verschiedene genetische Ansätze wie chemische Mutagenese, homologe Rekombination und Genom-Editierung eingesetzt. In den letzten Jahren wurden zahlreiche Mausmutagenese-Programme durchgeführt und in einigen Fällen wurden die genetisch veränderten embryonalen Stammzellen oder auch fertiggestellte Mausmutanten der Wissenschaft zu überschaubaren Kosten zur Verfügung gestellt (z. B. https://www.komp.org/, http:// www.mousephenotype.org/, https://www.infrafrontier.eu/search). Allerdings ist der Weg von den embryonalen Stammzellen zur homozygoten Mausmutante anspruchsvoll und auch von universitären HNO-Kliniken typischerweise nur in Zusammenarbeit mit einschlägig vorerfahrenen Kooperationspartnern zu leisten. Bei Interesse an der Funktion eines spezifischen Gens empfiehlt sich eine Recherche der an diesem Gen arbeitenden Gruppen, auch oder gerade wenn diese nicht selbst am Ohr forschen. Zudem bieten Firmen inzwischen die Erstellung solcher genetisch veränderter Tiere für Forschungszwecke an, doch die Kosten dort sind erheblich. Schließlich sind die Tierhaltungskapazitäten an zahlreichen Universitätsmedizin-Standorten begrenzt und/oder die Haltungskosten sehr hoch, sodass eine umfassende Analyse von Genen im Mausmodell dadurch limitiert wird. Ähnliche Repositorien existieren auch für mutante Fruchtfliegen und Zebrafische, deren Haltungskosten deutlich niedriger sind. Daher, aber auch durch einige Vorteile von Zebrafisch etwa bei der akuten Expression eines speziellen Gens, kann der Zebrafisch mit den Haarzellen seines Seitenlinienorgans und des Innenohrs einen wichtigen Zwischenschritt bilden (z. B. [6]). Man muss aber im Blick behalten, dass sich die Anatomie und Physiologie der Innenohren von Fischen und Säugetieren doch erheblich unterscheiden, sodass Aussagen zur humanen Schwerhörigkeit auf der Basis von Befunden am Zebrafisch mit großer Vorsicht getroffen werden sollten.

Die morphologische und funktionelle Charakterisierung benötigt die Verfügbarkeit zahlreicher Methoden wie Immunhistochemie, Licht- und Elektronenmikroskopie mit verschiedenem Auflösungsvermögen, systemische und zelluläre Hörphysiologie und Verhaltensanalysen. Einige der dabei verwendeten Methoden werden in den folgenden Kapiteln angesprochen. Die beobachteten Phänotypen geben Einsichten in die Rolle des Ziel-Gens beim Hören [5, 7]. Die an HNO-Kliniken vorhandene Expertise bei der morphologischen und funktionellen Analyse des Hörens wird sehr gern von Arbeitsgruppen aus anderen Feldern in Anspruch genommen. Dabei sind etwa Ableitungen von BERA und otoakustischen Emissionen an Nagern [8] ebenso wie die Immunhistochemie und Konfokalmikroskopie realisierbar und gleichzeitig geeignet für Doktorarbeiten und Forschungsrotationen von Weiterbildungsassistenten. Darüber hinaus können insbesondere systemphysiologische und verhaltensbiologische Befunde im Mausmodell einer genetischen Schwerhörigkeit sehr informativ für die klinische Diagnostik sein. So hat die Molekularphysiologie an der Maus zu einer pathophysiologischen Klassifikation der sensorineuralen Schwerhörigkeit geführt ( $>$ Abb. 2, $[7,9])$. Die Spezifität der beobachteten Phänotypen kann durch das Wiedereinbringen des gesunden Gens, z. B. mit viralen Vektoren, kontrolliert werden. Dabei können solche „Rettungsexperimente“ und ihre methodische Umsetzung im Mausmodell gleichzeitig als Machbarkeitsstudien für die Entwicklung möglicher gentherapeutischer Ansätze dienen [10-14]. Genetische Ansätze, die aufgrund angenommener Analogien zu anderen Systemen die Rolle von Kandidaten-Genen im Innenohr untersuchten, haben häufig interessante Abweichungen im molekularen Profil von Innenohrzellen gefunden und geholfen, alternative und unkonventionelle molekulare Mechanismen zu berücksichtigen (siehe etwa das Kapitel zur synaptischen Schallkodierung). Darüber hinaus waren diese Studien hilfreich bei der Etablierung von Methoden zur Untersuchung des Innenohrs. Schließlich sind die molekular- und zellbiologischen Einsichten eine wichtige Voraussetzung für Ansätze zur Haarzellregeneration [15] und anderen innovativen Therapieansätzen wie dem optischen Cochlea Implantat [16]. Auf dieser Grundlage wird in den USA aktuell bereits ein klinischer Versuch zur Transdifferenzierung von Stützzellen in Haarzellen durchgeführt [17], die durch viral-vermittelte Expression des Transkriptionsfaktors Atoh1 in diesen Zellen induziert werden soll [18]. Auch bei Strategien für die Zellersatztherapie mit aus Stammzellen gewonnenen exogenen Zellen gibt es weitere zellbiologische und tierexperimentelle Fortschritte wie etwa beim Ersatz von Spiralganglionneuronen (z. B. [19]). Die Möglichkeit Organoide des Innenohrs zu generieren [20-22], verspricht eine deutlich verbesserte Verfügbarkeit von Haarzellen und Nervenzellen für pharmakologische oder genetische Untersuchungen und Fortschritte für eine zukünftige Zellersatztherapie, für deren Entwicklung und potentielle klinische Translation aber sicher noch mehr als eine Dekade benötigt wird. Bis dahin bleiben Hörgeräte und Cochlea Implantate die wichtigsten Optionen der Hörrehabilitation und ihre weitere Verbesserung eine wichtige Aufgabe für Forschung und Entwicklung. Als ein zukunftsweisendes Beispiel dieser Aktivitäten soll hier die optische Stimulation der Cochlea genannt werden, in denen die deutsche Hochschulmedizin aktuell führend ist. Zum einen wird an der Medizinischen Hochschule Hannover und der Universitätsmedizin in Homburg/Saar durch die Möglichkeit mit Licht eine mechanische Reizung der Cochlea herbeizuführen die Entwicklung einer neuen Generation von Hörgeräten initiiert [23, 24]. Zum anderen wird an der Universitätsmedizin Göttingen in Zusammenarbeit mit Technologen der Universität Freiburg und der Technischen Universität Chemnitz ein optisches Cochlea Implantat zur optogenetischen Rei- 


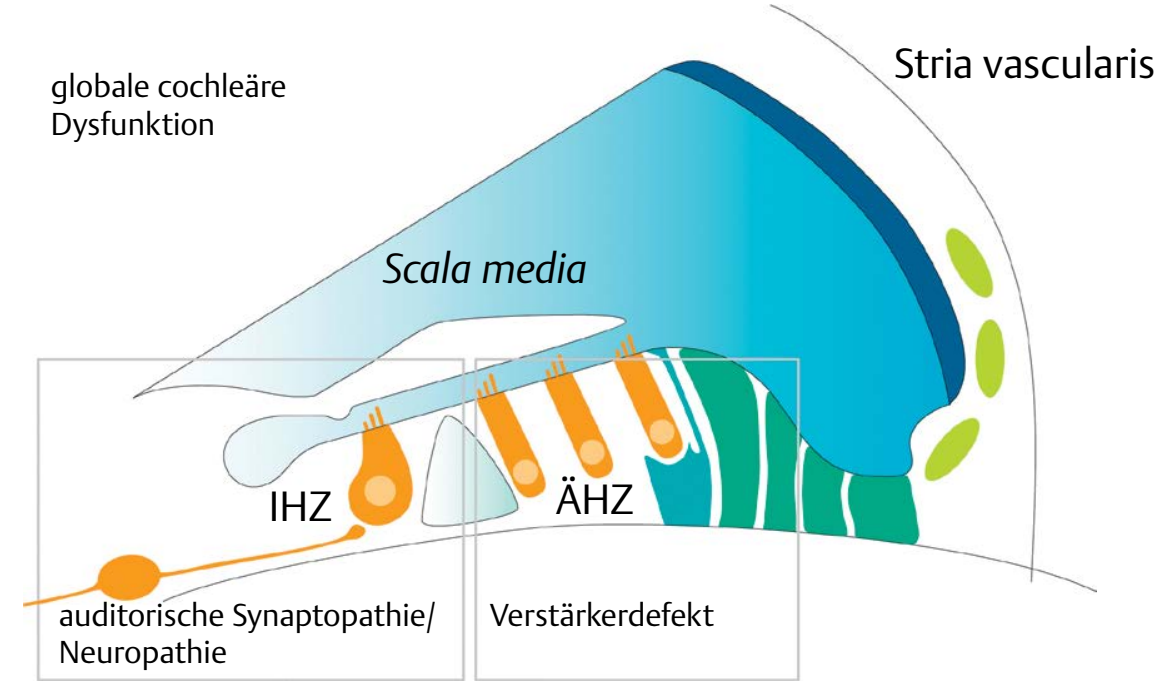

Abb. 2 Pathomechanismen der sensorineuralen Schwerhörigkeit. Schädigungen der äußeren Haarzellen bedingen eine Reduktion oder den Verlust der nichtlinearen cochleären Verstärkung. Dies beeinträchtigt die Sensitivität und Frequenzdiskrimination. Eine gestörte Schallkodierung und Reizweiterleitung durch die innere Haarzelle und Spiralganglionneurone führen zum Krankheitsbild der auditorischen Synaptopathie bzw. Neuropathie, bei dem v. a. die zeitliche Verarbeitung der aufgenommenen Schallreize gestört ist. Bei globalen cochleären Störungen wie z. B. gestörter Endolymphsekretion oder defekter mechanoelektrischer Transduktion sind sowohl die Verstärkermechanismen als auch die Schallkodierung beeinträchtigt (ÄHZ äußere Haarzellen, IHZ innere Haarzelle). Aus [9].

zung von viral-transduzierten Spiralganglionneuronen entwickelt $[25,26]$. Weil Licht besser gebündelt werden kann als Strom hat das optische Cochlea Implantat eine feinere Frequenzauflösung und verspricht so u. a. ein verbessertes Sprachverstehen im Störgeräusch.

\section{Der Kaliumkreislauf, die Transduktion und die Arbeitsteilung in der Cochlea}

Der sog. „Kaliumkreislauf“ des Innenohrs ist ein „Hotspot“ der genetischen Schwerhörigkeit, zu dem auch die Connexin 26 Gap Junction Kanäle gehören, die bei der häufigen autosomal rezessiven Schwerhörigkeit DFNB1A defekt sind. Die physiologische Signifikanz liegt bei der Verwendung von Kalium-Ionen für die mechanoelektrische Transduktion der Haarzellen. Dies ist ein kleiner evolutionärer Geniestreich, der den Haarzellen die elektrische Erregung (Rezeptorpotenzial) erlaubt, ohne dass metabolische Energie für die Wiederherstellung der Natrium- und Kalium-Homöostase aufgewandt werden muss. Anders als im Neuron, wo Natrium das erregende Ion darstellt und zum Wiederausgleich des Potenzials entgegen dem lonen-Gradienten im Austausch gegen Kalium wieder in den Extrazellulär-Raum gepumpt werden muss, öffnet die Haarzelle „einfach“ spannungsgesteuerte Kalium-Kanäle der basolateralen Membran und setzt passiv Kalium in die Perilymphe frei. Einer der dabei beteiligten Kalium-Kanäle ist KV7.4 (oder KCNQ4), welcher bei der autosomal dominanten Schwerhörigkeit DFNA2 defekt ist [27, 28]. Damit jedoch bei der mechanoelektrischen Transduktion Kalium aus der Endolymphe in die Haarzelle einströmen kann, benötigt es dort einen entsprechenden elektrochemischen Gradienten (treibende Kraft: hohe Kalium-Konzentration [ca. $150 \mathrm{mM}$ ] und positives endolymphatisches Potenzial [80-120 mV]), der stetig von der Stria vascula- ris erzeugt wird.

Auf diese Weise wird die mechanoelektrische Transduktion der Haarzelle, deren Sauerstoff-Versorgung bedingt durch die für maximale akustische Sensitivität minimierte Blutperfusion im Corti Organ schlecht ist, von metabolischer Last befreit, die vorwiegend von der Stria vascularis getragen wird („Arbeitsteilung“). Die Haarzelle nutzt für die mechanoelektrische Transduktion hochspezialisierte mechanosensitive Ionenkanäle, die bei Auslenkung der Stereocilia von einem feinen „Faden“ („tip-link“, > Abb. 3a) geöffnet werden. Aktuell gelten sog. Transmembrane like Channels (TMCs 1 und 2) als aussichtsreiche Kandidaten für den Haarzell-Transduktionskanal ( Abb. 3b, [29-31]). Diese Gene sind bei den humanen Schwerhörigkeiten DFNB7/11 und DFNA36 betroffen und kürzlich konnte in entsprechenden Mausmodellen mittels viralem Gen-Transfer die prinzipielle Machbarkeit einer Gen-Therapie gezeigt werden [12]. Die tip-links werden aus den extrazellulären Matrix-Proteinen Cadherin 23 und Protocadherin 15 gebildet [32] und durch raffinierte Proteinkomplexe in der Membran der Stereocilia verankert [5] ( Abb. 3a).

Mutationen einiger Gene für die Proteine der tip-links und die Verankerungsproteine führen zum Usher-Syndrom (sensorineurale Schwerhörigkeit, Retinitis pigmentosa je nach Typ mit vestibulärem Funktionsverlust)[5], da sie auch für die Funktion von Photorezeptoren des Auge wichtig sind. Nachdem Kalium der mechanoelektrischen Transduktion der Haarzellen gedient hat und von ihnen in die Perilymphe freigesetzt wurde, gelangt es vermutlich über mehrere Wege zurück in die Stria vascularis („Kaliumkreislauf“, > Abb. 3c). Man glaubt, dass „Stützzellen“ im Corti Organ, vergleichbar mit den Glia-Zellen des Gehirns, Kalium über Ionenpumpen wie KCC4 aufnehmen. Das Kalium diffundiert dann vermutlich über aus Connexin 26 und 30 (beide kodierenden Gene sind Taubheitsgene) gebildete 

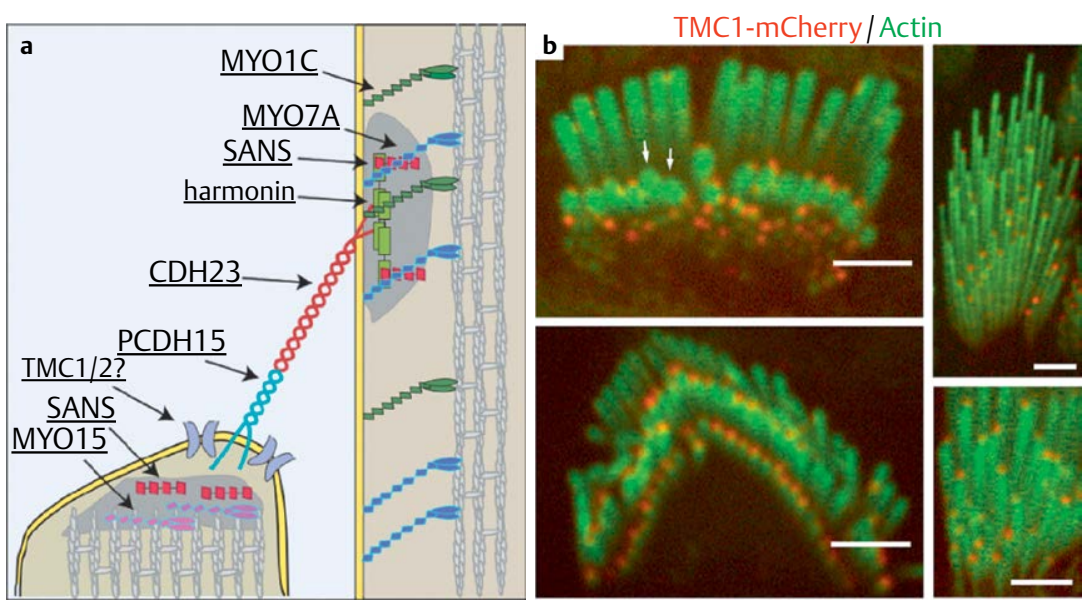

C
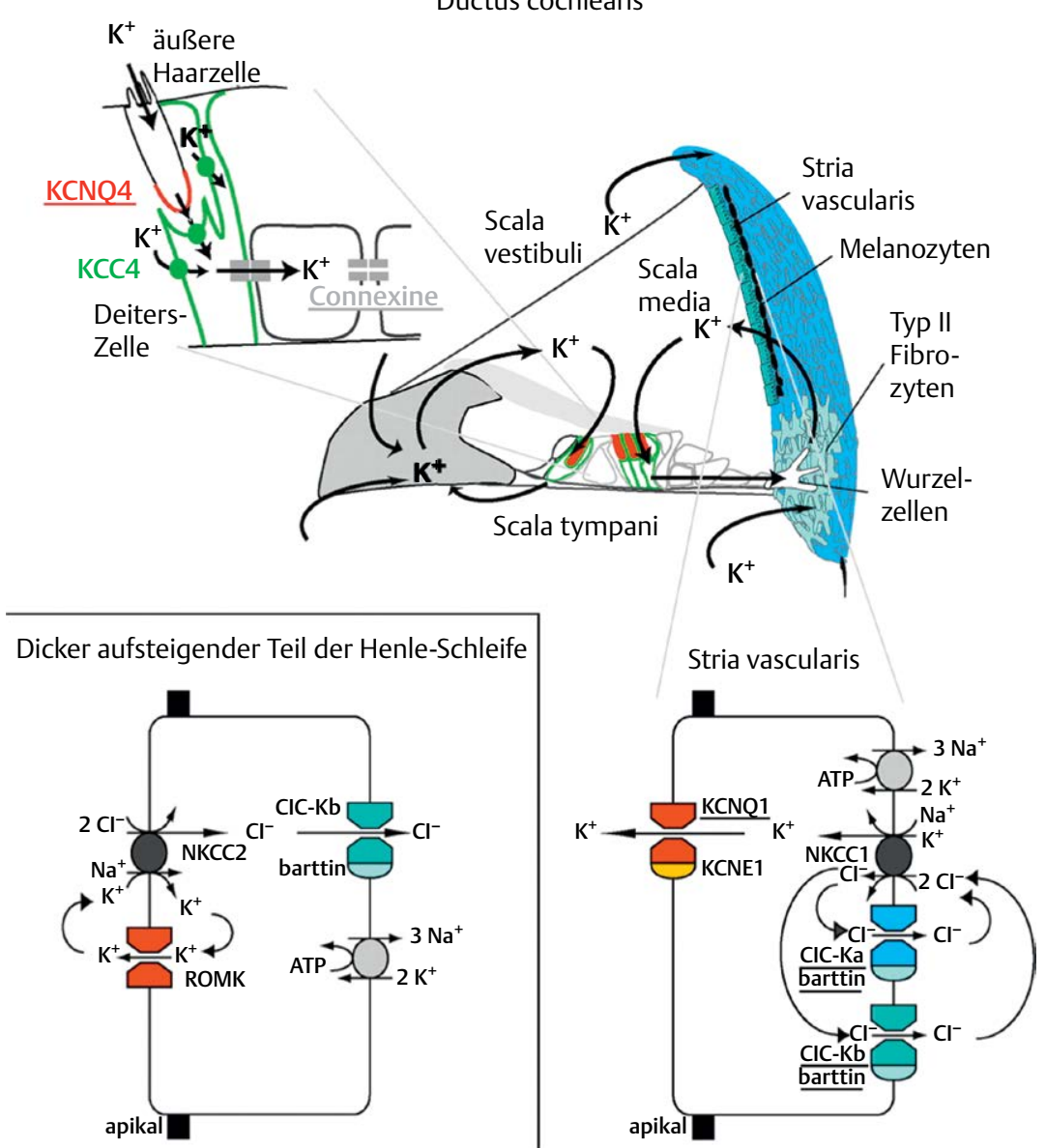

- Abb. 3 KaliumKreislauf in der Cochlea. a „Tip links“ in den Stereocilia der Haarzellen werden durch einen komplexen molekularen Apparat gebildet und verankert. Die daran beteiligten Proteine werden sämtlich von als Taubheitsgene identifizierten Sequenzen kodiert. b TMC1 ist ein Kandidat für den Kalium-durchlässigen Mechanotransduktionskanal der Stereocilia, welcher durch Zug an den „tip links“ geöffnet wird. Immunfluoreszenzfärbungen gegen markiertes TMC1-Protein (rot) lokalisieren es an den Spitzen der Stereocilia, welche durch Actin (grün) markiert werden. Die Bilder zeigen die Stereocilia von inneren Haarzellen (oben links), äußeren Haarzellen (unten links) und vestibulären Haarzellen (oben und unten rechts) der MausCochlea. Maßstabsbalken: $2 \mu \mathrm{m}$. Modifiziert aus [31]. c Der Kaliumkreislauf in der Cochlea ermöglicht die Aktivität der Haarzellen. Die hohe KaliumKonzentration in der Scala media führt zu Einstrom in die Haarzellen, aus welchen das Kalium u. a. über KCNQ4-Kanäle ausströmt. Dort wird es von „Stützzellen“ aufgenommen und über Gap Junction Kanäle weiter bis zur Stria vascularis geleitet, wo es über NKCC1 in die marginalen Zellen (unten rechts) aufgenommen wird. Von dort wird es über KCNQ1-Kanäle wieder in die Scala media ausgeschüttet. Auch an diesem Prozess sind zahlreiche Taubheitsgene (unterstrichen) beteiligt. Unten links zum Vergleich die ähnliche Funktionsweise der Kalium-Wiederverwertung in der Niere. Modifiziert aus [50]. 
Gap-Junction Kanäle durch das Netzwerk der „gekoppelten“ Stützzellen und in die ebenfalls durch Gap Junctions gekoppelten Fibrozyten der lateralen Wand der Cochlea, bis es schließlich vom Epithel der Stria vascularis „wiederverwendet wird“. Die Stria vascularis, ein „nieren-ähnliches“ Epithel in der lateralen Wand der Cochlea mit sehr effizienter Durchblutung („vascularis“), ist mit einem raffinierten System von lonenkanälen und lonenpumpen ausgestattet ( $>\mathbf{A b b} \mathbf{3} \mathbf{3 \mathbf { c }}$ ). Für einige der kodierenden Gene sind pathogene Mutationen bekannt, die zu Schwerhörigkeit und gelegentlich auch zu Nieren- oder Herzerkrankungen führen (syndromale Schwerhörigkeit). Defekte des Kaliumkreislaufs bzw. der Transduktion führen zu einer globalen cochleären Dysfunktion ( $\triangleright$ Abb. 2), da die treibende Kraft sowohl für äußere als auch für innere Haarzellen und deren Output-Funktion fehlt (siehe unten). Schließlich resultiert oft eine Degeneration der sensorischen Elemente der Cochlea.

\section{Die cochleäre Verstärkung und die synaptische Schallkodierung}

Die mechanoelektrische Transduktion führt zum Rezeptorpotenzial, das in den äußeren Haarzellen primär zur sog. „Elektromotilität“ und in den inneren Haarzellen zur Freisetzung des Botenstoffs Glutamat an den Synapsen mit den Typ-1 Hörnervenzellen führt. Die
Elektromotilität der äußeren Haarzellen (spannungsgesteuerte Veränderungen der Zelllänge) wird durch das sog. ,,Motorprotein“ Prestin (von Presto, schnell) vermittelt und bildet wahrscheinlich den Mechanismus der dem cochleären Verstärker zugrunde liegt [33, 34]. Prestin ist ein Protein, das bei Spannungsänderung seine Konformation ändert und kann vereinfacht als eine Art Piezo-Kristall dargestellt werden. Wie die Spannung in unseren Quartz-Uhren führt das Rezeptorpotenzial zu einer Änderung der Ausdehnung von Prestin in der seitlichen Haarzellmembran. Da sehr viele Prestin Moleküle quasi „in Serie“ dasselbe tun kommt es zu einer mikroskopisch sichtbaren Längenänderung der Haarzelle, die mechanische Energie in die Schwingung des Corti-Organs einführt. Selektive Dysfunktion durch Mutationen im Prestin-Gen [35] oder Verlust der Elektromotilität bzw. der äußeren Haarzellen [27, 28] führt zu einer Schwerhörigkeit die man physiologisch als „Verstärkerdefekt“ bezeichnen kann ( $\triangleright$ Abb. 2). Neben dem Verlust der durch die Elektromotilität der äuBeren Haarzellen bedingten otoakustischen Emissionen erwartet man hier einen Hörverlust von maximal 50-60 dB [36] und eine verschlechterte Frequenzdiskrimination [37].

In den inneren Haarzellen öffnet das depolarisierende Rezeptorpotenzial spannungsgesteuerte $\mathrm{Ca}$ 1.3 $\mathrm{Ca}^{2+}$-Kanäle an den aktiven Zonen der Transmitterfreisetzung. Das einströmende $\mathrm{Ca}^{2+}$ dient als Signal, das die mechanoelektrische Transduktion an die Freisetzung des Transmitters Glutamat koppelt, welcher in der postsynaptischen

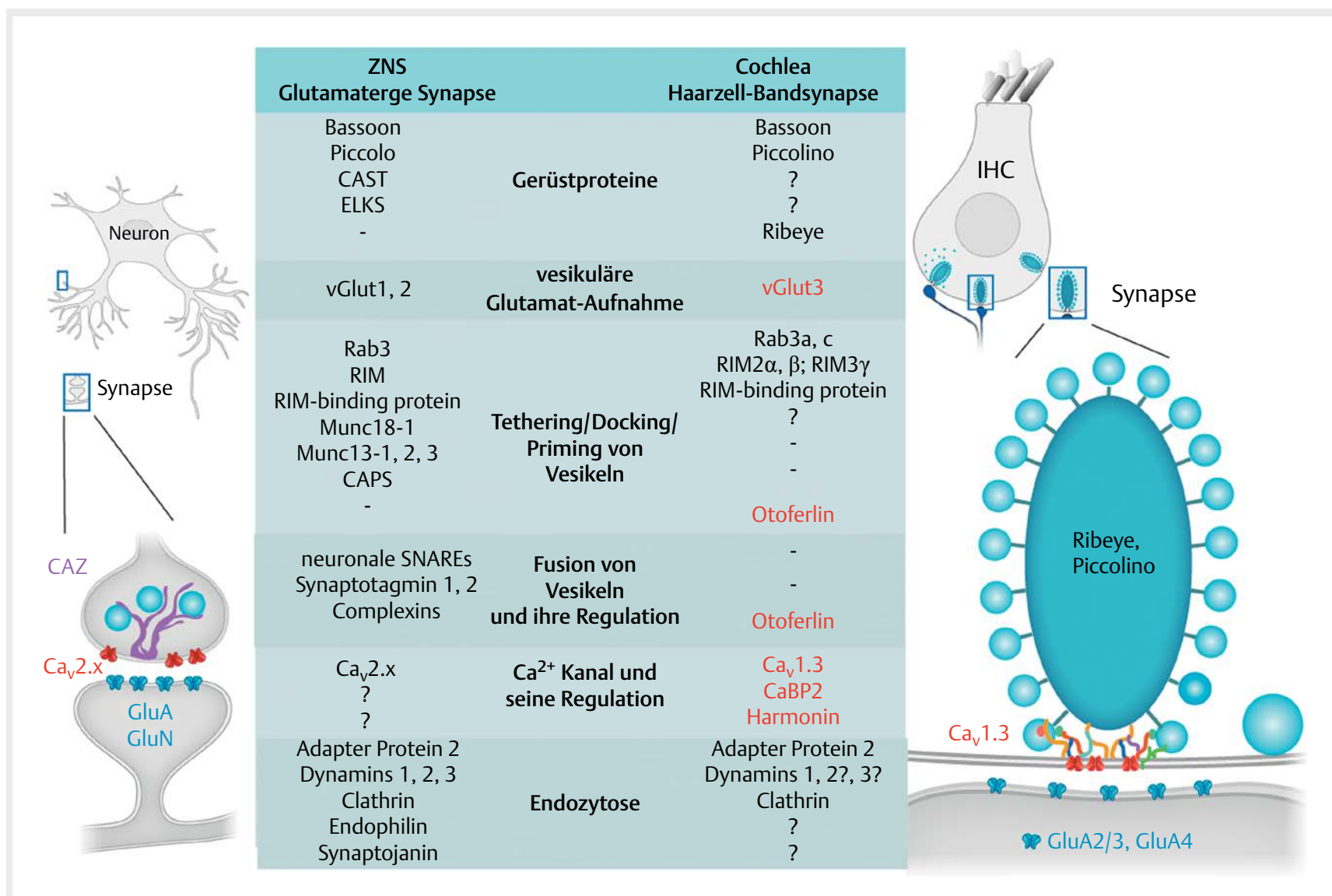

- Abb. 4 Vergleich von synaptischen Proteinen in „konventionellen“ glutamatergen Synapsen des zentralen Nervensystems und in Bandsynapsen der Cochlea. Zahlreiche Proteine aus konventionellen Synapsen (links) sind in Bandsynapsen der Haarzellen (rechts) offenbar nicht vorhanden, während es dort spezielle Proteine gibt, von denen etliche durch Taubheitsgene kodiert werden (rot markiert). Modifiziert aus [38]. 
Hörnervenzelle zur Erregung und schließlich zur Generierung eines Aktionspotenzials führt, das das Gehirn über den akustischen Reiz „informiert“. Die aktiven Zonen der inneren Haarzellen sind sehr spezialisiert um den hohen funktionellen Anforderungen der Schallkodierung an die Rate, zeitliche Präzision und Dauer der synaptischen Transmission zu genügen ( $\triangleright$ Abb. 4). Die Spezialisierung wird eindrücklich durch die Anwesenheit des aus dem Protein Ribeye gebildeten synaptischen Bands und die auch ansonsten von der „typischen“ glutamatergen Synapse des ZNS abweichende molekulare Maschinerie verdeutlicht. Für das Ziel dieses Übersichtsartikels sind dabei weniger die einzelnen Proteine (Übersichten in [38-40]) wichtig, sondern mehr das Ausmaß der Abweichung vom „konventionellen Bauplan“ und der Fakt, dass mehrere der für die Haarzellsynapse kodierende Gene bei erblicher Schwerhörigkeit betroffen sind [7,41]. Neben dem $\mathrm{Ca}_{\mathrm{v}} 1.3 \mathrm{Ca}^{2+}$-Kanal und seinem Regulator CaBP2 ist hier v. a. das Protein Otoferlin zu nennen, das offenbar eine Rolle als „Master-Protein“ der Haarzellsynapse trägt [7, 38]. Genetische Defekte der für die Haarzellsynapse kodierenden Gene führen zu einer Schwerhörigkeit die als auditorische Synaptopathie/Neuropathie bezeichnet wird ( $\triangleright$ Abb. 2). So führen Defekte im Otoferlin Gen zu auditorischen Synaptopathien (DFNB9 und temperaturempfindliche Schwerhörigkeit), bei denen die cochleäre Funktion upstream der Haarzellsynapse (zumindest initial) intakt sein kann, aber die zeitlich präzise Schallkodierung eingeschränkt oder unmöglich ist $[7,42]$. Dementsprechend findet man otoakustische Emissionen und/oder Mikrophon/Summationspotenziale (Elektrocochleografie), aber kein - oder ein pathologisches - Summenaktionspotenzial bzw. BERA. Subjektiv kann die Tonschwelle recht gut erhalten sein, das Sprachverstehen ist v. a. im Störschall jedoch sehr schlecht [7,42]. Auch im Bereich der synaptischen Gendefekte haben bereits erste Studien eine Wiederherstellung der Hörfunktion durch viralen Gen-Transfer im Tiermodell gezeigt $[10,43]$. Jedoch sind in diesem Bereich noch viele Fragen offen bevor man an eine klinische Umsetzung dieses Ansatzes gehen kann. Bereits jetzt kann aber festgehalten werden, dass die Ergebnisse der Hörrehabilitation mit Cochlea Implantaten bei Patienten mit auditorischen Synaptopathien sehr gut sind, während Hörgeräte nur unzureichenden Benefit für die Betroffenen liefern (z. B. DFNB9, [7]). Dagegen sind die Ergebnisse über verschiedene Formen der auditorischen Neuropathie hinweg weniger eindeutig [44]. Die bereits begonnenen Bemühungen zur Korrelation von molekulargenetischen Befunden und dem Outcome bei Cochlea Implantat Versorgung (z. B. [45]) verspricht dem HNO-Arzt verbesserte Möglichkeiten zur Beratung von hörgestörten Kandidaten für ein Cochlea Implantat.

Das Verständnis der Synaptopathie bzw. des Synapsenverlusts ist jedoch von genereller Bedeutung für die klinische Hals-Nasen-Ohrenheilkunde. Tierexperimente und Untersuchungen am menschlichen Felsenbein legen nahe, dass die Schädigung und der Verlust von Haarzellsynapsen auch bei häufigen Formen der Schwerhörigkeit, wie der Lärmschwerhörigkeit, der altersbegleitenden Hörstörung (komplex-genetische Erkrankungen: genetische Prädisposition und exogene Einflüsse) und der Schwerhörigkeit nach Einwirkung einiger ototoxischer Medikamente sowie beim Morbus Menière für das Krankheitsbild ursächlich ist [7, 46, 47]. Das klassische Experiment von Kujawa und Liberman [48] zum temporären Schwellenschwund in der Maus deutet darauf hin, dass ein Lärmtrauma, selbst bei kompletter Erholung der otoakustischen Emissionen und der Hörschwelle, zu einem irreversiblen Verlust von bis zur Hälfte aller
Haarzellsynapsen führen kann: „Hidden Hearing Loss“. Dies wurde mit Immunhistochemie für das synaptische Band [49] und BERA Messungen belegt, die eine lineare Beziehung zwischen der Amplitude des Summenaktionspotenzial des Hörnervs und der Zahl der Haarzellsynapsen zeigte. Inzwischen wurden vergleichbare Befunde auch an anderen Spezies erhoben und mittels Elektrocochleografie an Jugendlichen mit Lärmanamnese eine relative Verminderung des Summenaktionspotenzials gezeigt [46]. Weitere klinische Studien wie auch Analysen von humanen Felsenbeinen sollten die Übertragbarkeit der tierexperimentellen Befunde erhärten bzw. überprüfen.

\section{Zusammenfassung}

Die Fortschritte in der Genetik und Molekularphysiologie des Hörens ermöglichen ein besseres Verständnis der molekularen Mechanismen von Hören und sensorineuraler Schwerhörigkeit. Um diesen Fortschritt für die klinische Praxis zu nutzen, ergeben sich die Notwendigkeiten für Updates aus der aktuellen Forschung, für differenzierte audiometrische Diagnostik und für die Zusammenarbeit mit NGS-erfahrenen Humangenetikern. Zunächst geht es also um verbesserte Diagnostik, v. a. bei Verdacht auf genetische Hörstörung. Dies hilft der Beratung der Betroffenen und ihrer Familien und erlaubt die differenzierte Wahl der Hörrehabilitation: z. B. Hörgerät vs. Cochlea Implantat bei der auditorischen Synaptopathie. Ähnliches gilt auch für die differenzierte Diagnostik von häufigen Formen der sensorineuralen Schwerhörigkeit wie der Lärmschwerhörigkeit. Die Translation von innovativen pharmakologischen oder gentherapeutischen Therapieverfahren für die sensorineurale Schwerhörigkeit wird noch Jahre der Forschung erfordern, aber hier sind nun Fortschritte absehbar. Es empfiehlt sich also „auf Empfang zu bleiben“.

\section{Danksagung}

Ich möchte mich bei Dr. Jakob Neef für die Unterstützung bei der Erstellung der Abbildungen, bei Dr. Neef, Patricia Räke-Kügler, Profs. Dirk Beutner und Bernd Wollnik für wertvolles Feedback zum Manuskript, und Prof. Christian Kubisch für eine Vorlage für Abbildung 1 bedanken.

Interessenkonflikt

Der Autor gibt an, dass kein Interessenkonflikt besteht.

Literatur

[1] Petit C, Levilliers J, Hardelin JP. Molecular genetics of hearing loss. Annu Rev Genet 2001; 35: 589-646

[2] Duman D, Tekin M. Autosomal recessive nonsyndromic deafness genes: a review. Front Biosci Landmark Ed 2012; 17: 2213-2236

[3] Shearer AE, Smith RJH. Massively Parallel Sequencing for Genetic Diagnosis of Hearing Loss: The New Standard of Care. Otolaryngol-Head Neck Surg Off J Am Acad Otolaryngol-Head Neck Surg 2015; 153: $175-182$ 
[4] Atik T, Bademci G, Diaz-Horta O, Blanton SH, Tekin M. Whole-exome sequencing and its impact in hereditary hearing loss. Genet Res 2015; 97: e4

[5] Richardson GP, de Monvel JB, Petit C. How the genetics of deafness illuminates auditory physiology. Annu Rev Physiol 2011; 73: 311-334

[6] Vogl C, Panou I, Yamanbaeva G, Wichmann C, Mangosing SJ, Vilardi F, Indzhykulian AA, Pangršič T, Santarelli R, Rodriguez-Ballesteros M, Weber T, jung S, Cardenas E, Wu X, Wojcik SM, Kwan KY, Del Castillo I, Schwappach B, Strenzke N, Corey DP, Lin S-Y, Moser T. Tryptophan-rich basic protein (WRB) mediates insertion of the tail-anchored protein otoferlin and is required for hair cell exocytosis and hearing. EMBO J 2016

[7] Moser T, Starr A. Auditory neuropathy - neural and synaptic mechanisms. Nat Rev Neurol 2016; 12: 135-149

[8] Pauli-Magnus D, Hoch G, Strenzke N, Anderson S, Jentsch T], Moser T. Detection and differentiation of sensorineural hearing loss in mice using auditory steady-state responses and transient auditory brainstem responses. Neuroscience 2007; 149: 673-684

[9] Strenzke N, Pauli-Magnus D, Meyer A, Brandt A, Maier H, Moser T. Update zur Physiologie und Pathophysiologie des Innenohrs. HNO 2008; 56: 27-36

[10] Jung S, Maritzen T, Wichmann C, Jing Z, Neef A, Revelo NH, Al-Moyed $H$, Meese S, Wojcik SM, Panou I, Bulut H, Schu P, Ficner R, Reisinger E, Rizzoli SO, Neef J, Strenzke N, Haucke V, Moser T. Disruption of adaptor protein $2 \mu(\mathrm{AP}-2 \mu)$ in cochlear hair cells impairs vesicle reloading of synaptic release sites and hearing. EMBO J 2015; 34: 2686-2702

[11] Askew C, Rochat C, Pan B, Asai Y, Ahmed H, Child E, Schneider BL, Aebischer P, Holt JR. Tmc gene therapy restores auditory function in deaf mice. Sci Transl Med 2015; 7: 295ra108

[12] Landegger LD, Pan B, Askew C, Wassmer S], Gluck SD, Galvin A, Taylor R, Forge A, Stankovic KM, Holt JR, Vandenberghe LH. A synthetic AAV vector enables safe and efficient gene transfer to the mammalian inner ear. Nat Biotechnol 2017

[13] Pan B, Askew C, Galvin A, Heman-Ackah S, Asai Y, Indzhykulian AA, Jodelka FM, Hastings ML, Lentz JJ, Vandenberghe LH, Holt JR, Géléoc GS. Gene therapy restores auditory and vestibular function in a mouse model of Usher syndrome type 1c. Nat Biotechnol 2017

[14] Moser T. Gene therapy for deafness: How close are we? Sci Transl Med 2015; 7: $295 f s 28$

[15] Brigande JV, Heller S. Quo vadis, hair cell regeneration? Nat Neurosci 2009; 12: 679-685

[16] Moser T. Optogenetic stimulation of the auditory pathway for research and future prosthetics. Curr Opin Neurobiol 2015; 34: 29-36

[17] Identifier: NCT02132130. Safety, Tolerability and Efficacy for CGF166 in Patients With Bilateral Severe-to-profound Hearing Loss. Im Internet: ClinicalTrials.gov

[18] Izumikawa M, Minoda R, Kawamoto K, Abrashkin KA, Swiderski DL, Dolan DF, Brough DE, Raphael Y. Auditory hair cell replacement and hearing improvement by Atoh 1 gene therapy in deaf mammals. Nat Med 2005; 11: 271-276

[19] Chen W, Jongkamonwiwat N, Abbas L, Eshtan S], Johnson SL, Kuhn S, Milo M, Thurlow JK, Andrews PW, Marcotti W, Moore HD, Rivolta MN. Restoration of auditory evoked responses by human ES-cell-derived otic progenitors. Nature 2012; 490: 278-282

[20] Koehler KR, Nie ], Longworth-Mills E, Liu X-P, Lee J, Holt JR, Hashino E. Generation of inner ear organoids containing functional hair cells from human pluripotent stem cells. Nat Biotechnol 2017; 35: 583-589

[21] Liu X-P, Koehler KR, Mikosz AM, Hashino E, Holt JR. Functional development of mechanosensitive hair cells in stem cell-derived organoids parallels native vestibular hair cells. Nat Commun 2016; 7: ncomms 11508

[22] Koehler KR, Mikosz AM, Molosh Al, Patel D, Hashino E. Generation of inner ear sensory epithelia from pluripotent stem cells in 3D culture. Nature 2013; 500: 217-221
[23] Wenzel GI, Balster S, Zhang K, Lim HH, Reich U, Massow O, Lubatschowski H, Ertmer W, Lenarz T, Reuter G. Green laser light activates the inner ear. J Biomed Opt 2009; 14: 044007

[24] Schultz M, Baumhoff P, Maier H, Teudt IU, Krüger A, Lenarz T, Kral A. Nanosecond laser pulse stimulation of the inner ear - a wavelength study. Biomed Opt Express 2012; 3: 3332

[25] Hernandez VH, Gehrt A, Reuter K, Jing Z, Jeschke M, Mendoza Schulz A, Hoch G, Bartels M, Vogt G, Garnham CW, Yawo H, Fukazawa Y, Augustine G], Bamberg E, Kügler S, Salditt T, de Hoz L, Strenzke N, Moser T. Optogenetic stimulation of the auditory pathway. J Clin Invest 2014; 124: 1114-1129

[26] Goßler C, Bierbrauer C, Moser R, Kunzer M, Holc K, Pletschen W, Köhler K, Wagner J, Schwaerzle M, Ruther P, Paul O, Neef J, Keppeler D, Hoch G, Moser T, Schwarz UT. GaN-based micro-LED arrays on flexible substrates for optical cochlear implants. J Phys Appl Phys 2014; 47: 205401

[27] Kubisch C, Schroeder BC, Friedrich T, Lütjohann B, El-Amraoui A, Marlin $S$, Petit C, jentsch T]. KCNQ4, a novel potassium channel expressed in sensory outer hair cells, is mutated in dominant deafness. Cell 1999; 96: 437-446

[28] Kharkovets T, Dedek K, Maier H, Schweizer M, Khimich D, Nouvian R, Vardanyan V, Leuwer R, Moser T, Jentsch TJ. Mice with altered KCNQ4 K\&plus; channels implicate sensory outer hair cells in human progressive deafness. EMBO J 2006; 25: 642-652

[29] Pan B, Géléoc GS, Asai Y, Horwitz GC, Kurima K, Ishikawa K, Kawashima Y, Griffith AJ, Holt JR. TMC1 and TMC2 Are Components of the Mechanotransduction Channel in Hair Cells of the Mammalian Inner Ear. Neuron 2013; 79: 504-515

[30] Kawashima Y, Géléoc GSG, Kurima K, Labay V, Lelli A, Asai Y, Makishima T, Wu DK, Della Santina CC, Holt JR, Griffith AJ. Mechanotransduction in mouse inner ear hair cells requires transmembrane channel-like genes. J Clin Invest 2011. Im Internet: http://www.ncbi. nlm.nih.gov/pubmed/22105175

[31] Kurima K, Ebrahim S, Pan B, Sedlacek M, Sengupta P, Millis BA, Cui R, Nakanishi H, Fujikawa T, Kawashima Y, Choi BY, Monahan K, Holt JR, Griffith AJ, Kachar B. TMC1 and TMC2 Localize at the Site of Mechanotransduction in Mammalian Inner Ear Hair Cell Stereocilia. Cell Rep 2015; 12: 1606-1617

[32] Kazmierczak P, Müller U. Sensing sound: molecules that orchestrate mechanotransduction by hair cells. Trends Neurosci 2012; 35: 220-229

[33] Oliver D. Prestin. In: The Senses: A Comprehensive Reference. New York: Academic Press; 2008: 309-317. Im Internet: http://www. sciencedirect.com/science/article/B8SV0-4RD4KNB-1G/2/9074ccebb1 68117c7790bdc6ebd94932

[34] Dallos P, Fakler B. Prestin, a new type of motor protein. Nat Rev Mol Cell Biol 2002; 3: 104-111

[35] Liu XZ, Ouyang XM, Xia XJ, Zheng J, Pandya A, Li F, Du LL, Welch KO, Petit C, Smith RJH, Webb BT, Yan D, Arnos KS, Corey D, Dallos P, Nance WE, Chen ZY. Prestin, a cochlear motor protein, is defective in non-syndromic hearing loss. Hum Mol Genet 2003; 12: 1155-1162

[36] Ryan A, Dallos P. Effect of absence of cochlear outer hair cells on behavioural auditory threshold. Nature 1975; 253: 44-46

[37] Fettiplace R, Hackney CM. The sensory and motor roles of auditory hair cells. Nat Rev Neurosci 2006; 7: 19-29

[38] Pangršič T, Reisinger E, Moser T. Otoferlin: a multi-C2 domain protein essential for hearing. Trends Neurosci 2012; 35: 671-680

[39] Safieddine S, El-Amraoui A, Petit C. The auditory hair cell ribbon synapse: from assembly to function. Annu Rev Neurosci 2012; 35: 509-528

[40] Moser T, Vogl C. New insights into cochlear sound encoding. F1000Research 2016; 5: 2081 
[41] Moser T, Predoehl F, Starr A. Review of hair cell synapse defects in sensorineural hearing impairment. Otol Neurotol Off Publ Am Otol Soc Am Neurotol Soc Eur Acad Otol Neurotol 2013; 34: 995-1004

[42] Moser T, Strenzke N, Meyer A, Lesinski-Schiedat A, Lenarz T, Beutner D, Foerst A, Lang-Roth R, Wedel H, Walger M, Gross M, Keilmann A, Limberger A, Steffens T, Strutz J. Diagnostik und Therapie der auditorischen Synaptopathie/Neuropathie. HNO 2006; 54: 833-841

[43] Akil O, Seal RP, Burke K, Wang C, Alemi A, During M, Edwards RH, Lustig LR. Restoration of Hearing in the VGLUT3 Knockout Mouse Using Virally Mediated Gene Therapy. Neuron 2012; 75: 283-293

[44] Rance G, Starr A. Pathophysiological mechanisms and functional hearing consequences of auditory neuropathy. Brain J Neurol 2015; 138: $3141-3158$

[45] Shearer AE, Eppsteiner RW, Frees K, Tejani V, Sloan-Heggen CM, Brown C, Abbas P, Dunn C, Hansen MR, Gantz BJ, Smith RJH. Genetic Variants in the Peripheral Auditory System Significantly Affect Adult Cochlear Implant Performance. Hear Res 2017; 348: 138-142
[46] Liberman MC. Noise-induced and age-related hearing loss: new perspectives and potential therapies. F1000Research 2017; 6: 927

[47] Kujawa SG, Liberman MC. Synaptopathy in the noise-exposed and aging cochlea: Primary neural degeneration in acquired sensorineural hearing loss. Hear Res 2015

[48] Kujawa SG, Liberman MC. Adding insult to injury: cochlear nerve degeneration after "temporary" noise-induced hearing loss. J Neurosci 2009; 29: 14077

[49] Khimich D, Nouvian R, Pujol R, Tom Dieck S, Egner A, Gundelfinger ED, Moser T. Hair cell synaptic ribbons are essential for synchronous auditory signalling. Nature 2005; 434: 889-894

[50] Hübner CA, Jentsch T]. Ion channel diseases. Hum Mol Genet 2002; 11: 2435-2445 